\title{
Public Private Partnership in deutschen Kommunen
}

\section{Wolfgang Gerstlberger Karsten Schneider}

Die Privatisierung der Deutschen Bahn AG wird derzeit kontrovers diskutiert. Andere öffentlich-private Partnerschaften bzw. Teilprivatisierungen ziehen weniger öffentliches Interesse auf sich. Ausnahmen wie „Toll Collect“ bestätigen die Regel. Gleichsam im Schatten der Privatisierungsdiskussion sind diese Formen mittlerweile auch hierzulande alltäglich geworden. Daher ist es angezeigt, nach 20 Jahren Praxis öffentlich-privater Partnerschaften in Deutschland eine Zwischenbilanz zu ziehen.

\section{Einleitung: Public Private Partnership und Privatisierungsdiskussion}

Public Private Partnerships (PPP; öffentlich-private Partnerschaften) werden als Instrumente für besseres Regierungshandeln diskutiert. Die Erwartung ist, dass auf diese Weise öffentliche Dienstleistungen rascher und um ca. $10 \%$ bis $25 \%$ kostengünstiger erbracht werden als bei konventioneller öffentlicher Leistungserstellung (Christen 2007). Die britische Labour-Regierung hat PPP als Alternative zu den Privatisierungen der Thatcher-Ära konzipiert. Die öffentliche Hand wurde wieder ins Spiel gebracht, da die Wirkungen vollständiger (materieller) Privatisierungen von der neuen Administration kritisch gesehen worden waren (Wegener 2002; Weizsäcker et al. 2006). Die Strategie eines Mittelwegs zwischen materieller Privatisierung und Beibehaltung der öffentlichen Rechtsform ist in der Bundesrepublik von sozialdemokratischen Entscheidungsträgern in Bund, Ländern und Kommunen aufgegriffen worden.

\subsection{PPP ALS ALTERNATIVE ZU MATERIELLER PRIVATISIERUNG?}

Eine Arbeitsgruppe der SPD-Bundestagsfraktion sowie Ministerpräsidenten und Minister in Bundesländern mit SPD-Regierungsbeteiligung forcieren seit Mitte der 1990er Jahre PPP. Angesichts struktureller Haushaltsengpässe der öffentlichen Hand soll PPP auch in Deutschland private Investitionen in die Infrastruktur ermöglichen, ohne die politische Steuerung teilprivati- sierter Aufgaben vollständig aus der Hand zu geben (SPD-Bundestagsfraktion 2004; Napp 2004; Littwin/Schöne 2006). Das „Gesetz zur Beschleunigung der Umsetzung von Öffentlich Privaten Partnerschaften und zur Verbesserung gesetzlicher Rahmenbedingungen für Öffentlich Private Partnerschaften“ (2005) basiert auf den Vorarbeiten der PPP-Arbeitsgruppe der SPD-Bundestagsfraktion.

In Deutschland wird PPP sowohl als Alternative als auch Vorstufe zu vollständiger Privatisierung diskutiert. Für beides gibt es Anhaltspunkte. Weltweit mündet ein Teil der seit Beginn der 1980er Jahre initiierten PPP in Privatisierungen. Ein weiterer Teil besteht bisher stabil als PPP fort. Es sind darüber hinaus auch einige Fälle dokumentiert, in denen PPP auf mehr oder weniger spektakuläre Weise scheiterten (IPPR 2001). Noch ist es schwierig, eine abschließende Einschätzung abzugeben, da die Laufzeit vieler PPP auf 10 bis 30 Jahre angelegt ist. Doch angesichts der ökonomischen, politischen und sozialen Brisanz von PPP - je nach Interpretation steht die Legitimation und Qualität öffentlicher Dienstleistungen oder die Effizienz ihrer Erbringung auf dem Spiel - ist eine Bestandsaufnahme angezeigt.

\subsection{ZIELE DES BEITRAGS UND VERSTÄNDNIS VON PPP}

Bislang gibt es kein einheitliches Verständnis von PPP. Wir schlagen daher vor, Kooperationen als PPP zu bezeichnen, die folgende Charakteristika aufweisen (Gerstlberger/Schneider 2008, S. 19ff.):

- langfristige Zusammenarbeit mit gegenseitiger Abhängigkeit,

- gemeinsame Strategie und Ziele,
- einvernehmliche Teilung der identifizierten Risiken und

- eine, über eine Auftraggeber-/Auftragnehmerbeziehung hinausgehende Zusammenarbeit.

In der Literatur werden zwei Grundtypen von PPP unterschieden (Grabow et al. 2005; Budäus 2006). Institutionelle PPP sind auf Dauer angelegt. In der Regel wird als Rechtsform die GmbH gewählt. Wesentliche Absprachen enthält der Gesellschaftervertrag. Vertragliche PPP bezeichnen langfristige Vereinbarungen zwischen öffentlichen und privaten Akteuren. Die wichtigsten Varianten in deutschen Kommunen sind (1) Bereitstellungs-, (2) Konzessions- und (3) Betreiber- oder BOTModelle (Gerstlberger et al. 2006). Bei der erstgenannten Variante erhält z. B. die Kommune durch den privaten Partner die langjährige Bereitstellungsgarantie für Großgeräte (z. B. in Kliniken). Diese umfasst Planung, Installation, Betrieb, Wartung, Service, Reparatur und - als Besonderheit - die kontinuierliche technologische Wei-

Wolfgang Gerstlberger, Prof. Dr., Betriebswirt, Leiter des Studiengangs BWL, Internationales Hochschulinstitut Zittau. Arbeitsschwerpunkte: Innovationsmanagement, Industrielle Beziehungen, Öffentliche Betriebswirtschaftslehre. e-mail: gerstlberger@ihi-zittau.de Karsten Schneider, Dr., Politikwissenschaftler, Referatsleiter in der Forschungsförderung der Hans-Böckler-Stiftung, Düsseldorf. Arbeitsschwerpunkte: Industrielle Beziehungen, politische Steuerung, öffentlicher Sektor.

e-mail: Karsten-Schneider@boeckler.de 
terentwicklung. Das Spezifikum der zweiten Variante besteht darin, dass ein privater Konzessionär im Auftrag der Kommune eine gebührenpflichtige Dienstleistung erbringt und dafür Nutzungsgebühren als Vergütung erhält. Bau bzw. Errichtung der notwendigen Infrastruktur können sowohl in öffentlicher als auch in privater Verantwortung liegen. Die dritte Variante ist der Sammelbegriff für unterschiedliche PPPModelle: Der private Betreiber übernimmt mindestens den Bau bzw. die Errichtung („build“), den Betrieb („operate“) und übergibt die kommunale Infrastruktureinrichtung an den öffentlichen Partner nach Vertragsende („transfer“). Der Private kann zusätzlich Planungs-, Finanzierungsbzw. Erhaltungsleistungen anbieten.

\subsection{ANFÄNGE DER DEUTSCHEN PPP-ENTWICKLUNG}

Auf der Bundes- und Landesebene dominieren seit Mitte der 1980er Jahre strategische Großprojekte. Beispiele dafür sind „Toll Collect“ (Lkw-Maut), „Herkules“ (Informations- und Kommunikationsinfrastruktur der Bundeswehr), „Galileo" (Satellitennavigation), „Expo 2000“ (Weltausstellung), „Stuttgart 21“ (Modernisierung des Bahnknotens Stuttgart), „MediaPark Köln“, „Internationale Bauausstellung (IBA) Emscher Park“, die Verkehrsprojekte „Herrentunnel“ (Lübeck) und „Warnowquerung“" (Rostock) oder „Güterverkehrszentren (GVZ)“ (z. B. in Kassel und Wolfsburg). Bei diesen Großprojekten und in der Frühphase der Etablierung von PPPs in deutschen Kommunen - bis Mitte der 1990er Jahre - waren mögliche Kostenund Zeiteinsparungen von geringer Bedeutung. Strategische Ziele, wie z. B. der Aufbau neuer lokaler bzw. regionaler Kooperationsnetzwerke oder die Mobilisierung privaten Know-hows, sind von kommunalen Entscheidungsträgern in dieser Phase höher gewichtet worden als mögliche Kostenund Zeiteinsparungen. Angesichts zunehmender Haushaltsengpässe der öffentlichen Hand wird PPP ab der zweiten Hälfte der 1990er Jahre auch in Deutschland verstärkt als Finanzierungsoption wahrgenommen, um den „Investitionsstau“ im Bereich der öffentlichen Infrastruktur zu verringern.

Eine wichtige Erfahrung aus dem britischen Infrastruktur-Programm Private Finance Initiative (PFI) ist, dass der Erfolg von PPP nur in einer langfristigen zeitli- chen Perspektive von mindestens zehn bis 15 Jahren beurteilt werden kann. Für Deutschland ist eine derartige Bewertung von PPP-Vorhaben bisher nur in Einzelfällen möglich, die bereits eine entsprechende Laufzeit aufweisen. Trotz dieser strukturellen Einschränkung liegt ausreichend empirisches, vor allem qualitatives Material für eine Zwischenbilanz kommunaler PPPs vor. In diesem Zusammenhang sind Veränderungen der rechtlichen und volkswirtschaftlichen Rahmenbedingungen in der Europäischen Union (EU) zu beachten.

\section{Rahmenbedingungen für PPP in der EU und in Deutschland}

\subsection{AKTUELLE ENTSCHEIDUNGEN AUF EU-EBENE}

Eine wichtige Einschränkung für öffentlich-private Partnerschaften in der EU betrifft „Inhouse-Geschäfte“. In einer Entscheidung vom 11.01.2005 (AZ C-26/03) hat der Europäische Gerichtshof (EuGH) festgestellt, dass Kommunen auch mehrheitlich von ihnen beherrschte Beteiligungen nicht ohne Ausschreibung beauftragen dürfen (EuGH 2005). Die öffentliche Hand hat ein Interesse daran, eigene bzw. ihr verbundene Gesellschaften mit der Aufgabenerledigung zu betrauen, da sie dadurch einen besseren Zugriff auf die Dienstleistungsqualität hat. Institutionelle PPP werden aufgrund der Rechtslage in deutschen Landkreisen und Gemeinden nur noch in Ausnahmefällen neu initiiert (z. B. Gesellschaften für Wirtschaftsförderung oder Tourismus). Bei den genannten Einsatzfeldern für PPP handelt es sich um freiwillige Selbstverwaltungsaufgaben. In diesen Aufgabenbereichen werden im Regelfall von PPP-Gesellschaften keine Leistungen für die kommunalen Gesellschafter erstellt, sondern Dienstleistungen für Dritte.

Eine weitere wichtige Rahmenbedingung für PPP in der EU ist die „EUROSTAT-Entscheidung“ (2004). Das statistische Amt der Europäischen Union hat sich in dieser Entscheidung mit der Bedeutung der Vermögenswerte in PPP vor dem Hintergrund der „Maastricht-Kriterien“ befasst. Aus Sicht der volkswirtschaftlichen Gesamtrechnung sollte ein Bewertungsmaßstab dafür gefunden werden, wie diese
Vermögenswerte sich auf die öffentliche Verschuldung in der EU auswirken.

Die EUROSTAT-Entscheidung zu PPP, die auf komplexen Modellen der volkswirtschaftlichen Gesamtrechnung basiert, lautet: „EUROSTAT empfiehlt, Vermögenswerte, die Gegenstand einer Öffentlich Privaten Partnerschaft sind, nicht als Vermögenswerte des Staates zu klassifizieren und folglich nicht in der Bilanz des Sektors Staat $\mathrm{zu}$ verbuchen, wenn die beiden folgenden Bedingungen erfüllt sind: [...D] er Private Partner trägt das Baurisiko, und [...] der Private Partner trägt mindestens entweder das Ausfallrisiko oder das Nachfragerisiko. Wenn das Baurisiko vom Staat getragen wird oder wenn der Private Partner nur das Baurisiko und kein anderes Risiko trägt, werden die Vermögenswerte als Vermögenswerte des Staates klassifiziert" (EUROSTAT 2004).

Diese Regelung soll einer etwaigen verdeckten Überschuldung öffentlicher Gebietskörperschaften vorbeugen, die als langfristige Konsequenz von Public Private Partnership eintreten könnte. Auslöser für die EUROSTAT-Entscheidung waren Untersuchungen von Rechnungshöfen und vergleichbaren Einrichtungen der Finanzaufsicht in verschiedenen EU-Mitgliedstaaten. Diese Evaluierungen dokumentierten Fälle, in welchen Kommunen aufgrund von PPP-Vorhaben ihre wirtschaftliche Leistungsfähigkeit überschritten hatten. Die operative Umsetzung der EUROSTATEntscheidung ist bisher in den EU-Mitgliedstaaten kaum vollzogen worden. Dies gilt auch für die Bundesrepublik. Der Bund und die Bundesländer haben eine entsprechende Konkretisierung ihrer Haushaltsbzw. Gemeindeordnungen bisher erst in Ansätzen umgesetzt (z. B. Gesprächsrunde PPP 2006, S. 30ff.).

\subsection{KRITISCHE EINSCHÄTZUNG DER RECHNUNGSHÖFE}

Die Konferenz der Präsidentinnen und Präsidenten der Rechnungshöfe des Bundes und der Länder hat 2006 auf die mit PPP-Finanzierungsmodellen verbundenen Überschuldungsgefahren dezidiert hingewiesen (Konferenz der Präsidentinnen und Präsidenten der Rechnungshöfe des Bundes und der Länder 2006):

„Städten, Gemeinden und auch Ländern, die finanziell angeschlagen sind, hilft dieses Instrument nicht weiter. Bei Finanzierungsengpässen wird es von der öffent- 
lichen Hand häufig als Ausweg angesehen, um Investitionen zu realisieren und Wachstumsimpulse zu setzen. Mittel- und langfristig ein gefährlicher Weg, weil auch hier die Finanzierungslast in die Zukunft verschoben wird [...]."

Hinweise aus unterschiedlichen Jahresberichten von Landesrechnungshöfen unterstreichen diese allgemeine Warnung. Im Jahresbericht des Bayerischen Obersten Rechnungshofes (OHR) (2006, S. 53) findet sich z. B. folgende Einschätzung: „Der Staat verwirklicht zwei Staatsstraßenprojekte über Öffentlich Private Partnerschaften. Der ORH hat bei den Investitionen keine Kostenvorteile festgestellt, die nicht auch bei konventioneller Verwirklichung erreichbar wären. Demgegenüber verteuert die private Vorfinanzierung die Maßnahmen." Der Präsident des Rechnungshofes Baden-Württemberg (Kiefer 2006) gelangt zu einer differenzierteren Einschätzung der Wirtschaftlichkeit von PPP, die in den 1990er Jahren realisiert wurden. Der explizite Hinweis auf Verschuldungsrisiken findet sich jedoch auch in diesem Bericht (ebd., S. 21).

Als wichtigste Erklärungsversuche dafür, dass die in PPP-Leitfäden (Weber et al. 2006; Christen 2007) häufig genannten Einsparpotenziale von ca. $10 \%$ bis $25 \%$ bei den Investitionskosten für die öffentliche Hand in der Praxis in der Regel erreichbar sind, lassen sich zusammenfassen (Gerstlberger/ Schneider 2008; Ziekow/Windoffer 2008):

- Hohe Transaktionskosten für die Projektvorbereitung und -begleitung durch externe Berater,

- nicht ausreichend qualifizierte Experten innerhalb der Verwaltung für Projektvorbereitung, -management und -controlling, - bislang unzureichende Standardisierbarkeit von PPP-Projekten hinsichtlich Leistungsspezifikation, Ausschreibungsund Vergabeprozessen sowie Vertragsgestaltung und

- begrenzte Realitätsnähe bisher verfügbarer Konzepte für öffentlich-private Wirtschaftsvergleiche.

Der erste und der dritte Punkt hängen auch damit zusammen, dass PPP nach wie vor ein Finanzierungs- und Beschaffungsinstrument ist, das in deutschen Gebietskörperschaften selten eingesetzt wird. Die öffentliche sowie private Infrastruktur für Beratung und Unterstützung ist dement- sprechend noch wenig entwickelt. Beratungsleistungen sind in der Regel projektspezifisch ausgerichtet und daher kostenintensiv. Eine stärkere Verbreitung und Standardisierung von PPP-Projekten könnte zu einer Verringerung der Transaktionskosten beitragen.

Was den letzten Punkt angeht, sind grundsätzliche methodische Probleme nach wie vor ungelöst. Das wahrscheinlich schwerwiegendste operative Problem besteht derzeit darin, dass betriebswirtschaftliche Instrumente (Doppik, Kosten- und Leistungsrechnung, Controlling) innerhalb des öffentlichen Sektors oft nicht ausreichend implementiert sind. Dies gilt besonders für kleine und mittlere Kommunen (Gerstlberger/Schmittel 2004). Die eigentlich für aussagekräftige Wirtschaftlichkeitsvergleiche mit den privaten Kalkulationswerten notwendigen öffentlichen Vergleichsdaten stehen daher häufig (noch) nicht zur Verfügung. Als Ersatz werden Durchschnittswerte (z. B. im Bereich des Immobilienmanagments) oder Schätzwerte verwendet, was jedoch zu erheblichen Ungenauigkeiten führt.

Ein weiteres Problem ist, dass langfristige Lebenszyklus-Risiken - besonders bei Vertragslaufzeiten bis zu 30 oder 35 Jahren - nur bedingt quantifiziert werden können. In vielen Fällen fehlen hier noch belastbare Erfahrungswerte. Besonders bei „HighTech-PPP“wie „Toll Collect“ müssen neben den herkömmlichen Projektrisiken zusätzlich technologische Innovationsrisiken berücksichtigt werden (Gerstlberger et al. 2006). Diese Entwicklungsrisiken werden in den bisher verfügbaren Standard-Kalkulationsinstrumenten für öffentlich-private Wirtschaftlichkeitsvergleiche aufgrund ihrer Komplexität nicht berücksichtigt.

Bis dato nicht geklärt ist darüber hinaus die Frage, wie umsatzsteuerpflichtige öffentlich-private Gesellschaften, die für den privaten Partner einen Gewinn erwirtschaften müssen, substanziell bessere Ergebnisse erreichen können als nicht umsatzsteuerpflichtige Eigen- oder Regiebetriebe (kommunale Einrichtungen in öffentlichen Rechtsformen), die nur kostendeckend arbeiten dürfen. Weitere offene Fragen betreffen die (fehlende) Möglichkeit, Fördermittel bzw. Zuschüsse für PPPs zu gewähren („Beihilfeproblematik“), oder Datenschutzanforderungen (Sühnel 2008; Gerstlberger/Sack 2003, S. 58).

In der Praxis werden Defizite des Beteiligungsmanagents und -controllings kaum offen diskutiert (Killian et al. 2006). Neuere empirische Studien (Edeling et al. 2004; Killian et al. 2006) konstatieren übereinstimmend ein „Steuerungsproblem“ (Killian et al. 2006, S. 125ff.) kommunaler Eigengesellschaften mit privaten Rechtsformen (in der Regel umsatzsteuerpflichtige Gesellschaften mit beschränkter Haftungen oder Aktiengesellschaften mit privater Beteiligung und mit Gewinnerzielungsabsicht sowie eigenständiger, dezentraler Möglichkeit der Kreditaufnahme):

- Die Kommune nimmt nicht angemessen auf ihre Beteiligungen Einfluss. Die Erfüllung des öffentlichen Zwecks wird zu wenig beachtet.

- Die Beteiligungsverhältnisse werden zunehmend komplexer und damit häufig unüberschaubar.

- Kommunale Haushalte drohen - auch aufgrund der Kreditaufnahmen von Eigengesellschaften - zu überschulden und es besteht die Gefahr, dass der zukünftige finanzielle Gestaltungsspielraum eingeschränkt wird.

- Bei Langzeitrisiken entstehen Vorsorgedefizite.

- Es kommt zu Koordinationsproblemen innerhalb der Gesamtverwaltung und zwischen unterschiedlichen Einzelgesellschaften (z. B. untereinander nicht abgestimmte Beschaffungs- oder EDV-Systeme).

Bemerkenswert ist zudem, dass diese Steuerungsprobleme bereits für privatrechtliche Töchter, die sich zu 100 \% im öffentlichen Eigentum befinden, konstatiert werden. Geschäftsführungen und Vorstände bemühen sich in der Regel aktiv, die Einflussnahme der Verwaltung und kommunaler Selbstverwaltungsorgane auf ein Mindestmaß zu reduzieren. Private Unternehmen sind demgegenüber eher in der Lage, ihre Interessen gegenüber ihren Tochtergesellschaften durchzusetzen (Schneider 2002).

\subsection{BEGRENZTE CHANCEN FÜR EINE "MITTELSTANDSFREUNDLICHE GESTALTUNG “}

Ein weiterer kritischer Hinweis zu PPP betrifft neben dem Verschuldungsrisiko für die Gebietskörperschaften die geringen Chancen für mittelständische (Bau-) Unternehmen, sich als private Partner federführend zu beteiligen. In der Studie „PPP und Mittelstand“ des Deutschen Instituts für Urbanistik (Difu) wurden 30 PPP- 


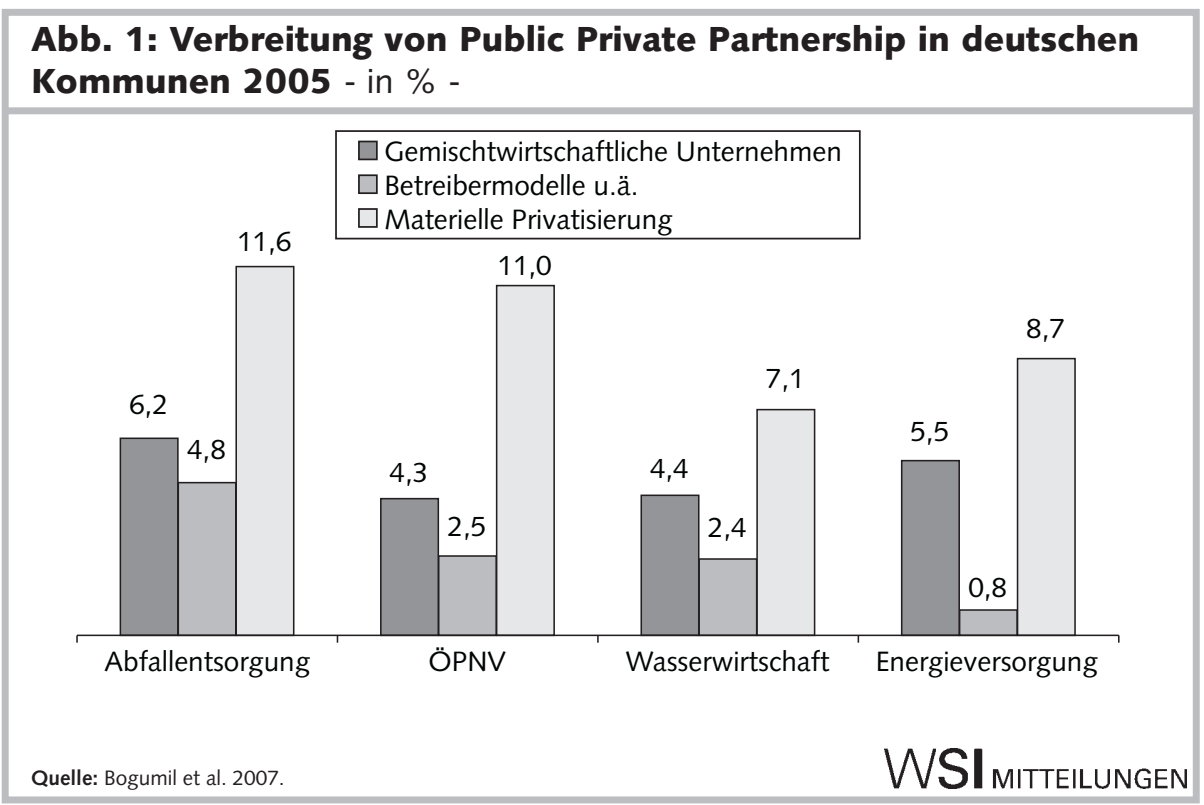

Hochbauprojekte hinsichtlich der Möglichkeiten einer „mittelstandsfreundlichen Ausgestaltung" untersucht (Difu 2008, S. 8). Ein Ergebnis dieser Studie lautet: „In großen Projekten finden sich praktisch keine KMU mehr als Hauptauftragnehmer, auch nicht als Partner in Konsortien." Ein rein mittelständisches Bieterkonsortium wurde in keinem der untersuchten PPPProjekte als privater Partner ausgewählt. Großunternehmen waren bei allen analysierten Projekten beteiligt, zumindest über Tochtergesellschaften (ebd., S. 9).

\section{Quantitative Verbreitung von PPP in deutschen Kommunen}

\subsection{PPP-VORGÄNGERMODELLE IN DER KOMMUNALEN VER- UND ENTSORGUNG}

Zu Beginn der 1980er Jahre förderte die niedersächsische Landesregierung im Rahmen der Neuordnung von $\$ 149$ Abs. 6 des Niedersächsischen Wassergesetzes Betreibermodelle unter der Beteiligung privater Entsorgungsunternehmen. Betreiberverträge konnten für einen Zeitraum von ca. 20 bis 30 Jahren abgeschlossen werden. Wichtigstes Ziel der privaten Beteiligung war es, angesichts nicht ausreichender Rückstellungen Investitionsbedarfe in den Kommunen zu verringern. Ähnliche Modelle sind nach der deutschen Vereinigung in den neuen deutschen Bundesländern zur führte 1989 und 1990 das Institut für Landes- und Stadtentwicklungsforschung GmbH (ILS) in NRW durch. 235 oder $59 \%$ von 396 angefragten Kommunen nahmen teil. Bei der Grundgesamtheit handelte es sich um alle Mitglieder des Deutschen Städtetags in NRW (Kruzewicz 1993). Für 103 Gemeinden konnten 286 öffentlichprivate Kooperationen ermittelt werden. 70 davon waren eine $\mathrm{GmbH}$ oder ein eingetragener Verein. Die Mehrzahl ließ sich den Aufgabenfeldern Stadtentwicklung und Technologieförderung zuordnen.

Im Rahmen einer repräsentativen Befragung von (Ober-)Bürgermeistern (aller Gemeindegrößenklassen) und Landräten aus dem gesamten Bundesgebiet wurde die Verbreitung von PPP für 2005 in ausgewählten Aufgabenbereichen ermittelt ( $A b$ bildung 1; Bogumil et al. 2007, S. 338).

Betreibermodelle (vgl. Abschnitt 1.2) spielen demnach lediglich in der Abfallentsorgung mit fast $5 \%$ aller Fälle eine nennenswerte Rolle (Anteil gemeinwirtschaftlicher Unternehmen mit Gesellschafteranteilen in öffentlicher und privater Hand: 6,2\%). Im Öffentlichen Personennahverkehr (ÖPNV), in der Wasserwirtschaft und der Energieversorgung erreicht der Anteil öffentlich-privater Unternehmen jeweils (knapp) die 5-\%-Marke. Der Anteil vollständiger (materieller) Privatisierung reicht dagegen von 7,1 \% bei der Wasserversorgung und Abwasserentsorgung bis 11,6\% im ÖPNV.

\section{3 ÖFFENTLICHER HOCHBAU ALS AKTUELLER PPP-SCHWERPUNKT}

Seit Ende der 1990er Jahre liegt der Schwerpunkt für PPP in deutschen Kommunen im öffentlichen Hochbau. Das Difu ermittelte für 2000 bis 2005 ,etwa 600“ deutsche PPP-Projekte, ,im weiteren Sinne (nach der Einschätzung der Befragten)“. Der kommunalen Ebene lassen sich „davon etwa 80 Prozent" zurechnen (Grabow et al. 2005, S. 4). $46 \%$ der identifizierten PPP entfallen auf den öffentlichen Hochbau. Die Vergleichswerte für Sport, Freizeit und Touristik sowie öffentliche Sicherheit und Justiz betragen $21 \%$ bzw. $12 \%$. In weiteren Feldern liegt der PPP-Anteil deutlich unter $10 \%$. Grabow et al. (2005) kommen darüber hinaus in einer Vollerhebung in Bund, Ländern und Gemeinden zu dem Ergebnis, dass sich die Zahl der realisierten und konkret geplanten kommunalen PPP zwischen 2004 und 2005 gegenüber den 
Vorjahren verdoppelt hat. Ausgenommen waren bei der Erhebung Stadtwerke sowie andere Ver- und Entsorgungsbetriebe.

Die Ergebnisse einer Befragung des Deutschen Städtetages aus dem ersten Halbjahr 2002 fallen ähnlich aus: $\mathrm{Zu}$ Beginn des neuen Jahrtausends war PPP in rund der Hälfte der 235 Mitgliedsstädte (Rücklaufquote $80 \%$ ) verbreitet. $53 \%$ der Mitgliedsstädte führten (nach Einschätzung der Befragten) im Befragungszeitraum „PPPbzw. PFI-Projekte" durch. Städte zwischen 100.000 und unter 200.000 (62 \%) sowie ab 500.000 Einwohnern (59\%) waren dabei im Vergleich zu den befragten Kommunen überdurchschnittlich aktiv. Die regionale Streuung ist erheblich:Während $100 \%$ der hessischen Städte Erfahrungen mit PPP gemacht haben, gilt dies lediglich für 33\% der Mitgliedsstädte in NRW (Frischmuth 2004, S. 44f.).

Die Kernergebnisse der Untersuchung von Janetschek (2007, S. 4ff.) bestätigen die Befunde der Difu-Studie im Wesentlichen. $17 \%$ der befragten Mittel- und Großstädte führen bereits Public Private Partnerships durch. $16 \%$ planen derzeit die Realisierung zumindest eines Projektes als PPP. Knapp die Hälfte (48 \%) der abgeschlossenen Projekte entfallen auf den Schulbereich (Hochbau). Danach folgen Sport, Freizeit und Kultur $(27 \%)$ sowie das Sozial- und Gesundheitswesen (ca. $18 \%$ ) als Anwendungsfelder. Die Bedeutung des Schulbereichs soll bis 2013 als PPP-Anwendungsfeld allerdings deutlich an Bedeutung verlieren: Nur noch 7 \% der Kommunen, die aktuell PPP-Vorhaben planen, nennen Schulprojekte als Option. Der Vergleichswert für das Feld Sport, Freizeit und Kultur beträgt $32 \% .80 \%$ der Befragten bewerten die „bisherigen Ergebnisse“ bzw. den Verlauf der bei ihnen durchgeführten Projekte „insgesamt positiv“ (Janetschek 2007, S.4), $4 \%$ geben „sehr negative Erfahrungen“ an. (ebd.). Die Kritik betrifft dabei PPP in der Summe etwas stärker als Privatisierungen.

Diese im Auftrag der Ernst \& Young AG ermittelten Befragungsergebnisse basieren auf 300 Telefoninterviews mit Kämmerern oder leitenden Mitarbeitern der Finanzverwaltungen deutscher Städte ab 20.000 Einwohnern. Die Interviews wurden im Mai 2007 durchgeführt. Bei der Interpretation der erhobenen Daten sind vier Einschränkungen zu berücksichtigen:

- Die Befragten beurteilen auch bestimmte Ergebnisse ihrer eigenen Aktivitäten.
- Städte mit unter 50.000 Einwohnern sind in der Stichprobe gegenüber der Grundgesamtheit unterrepräsentiert und solche mit zwischen 50.000 und unter 100.000 sowie zwischen 100.000 und unter 200.000 Einwohnern überrepräsentiert (Janetschek 2007, S. 5).

- Zwischen Privatisierungen und PPP sowie durchgeführten und geplanten Vorhaben wird in einigen Fragen nicht eindeutig unterschieden (z. B. Janetschek 2007, S. 31: Erfragt werden „Gründe für die geplanten oder bereits durchgeführten Privatisierungen bzw. ÖPP-Projekte").

- Die bisher - überwiegend 2004 oder 2005 - begonnenen PPP befinden sich zumeist in einem frühen Projektstadium.

\section{Zentrale Ergebnisse qualitativer Fallstudien zu PPP}

\subsection{DATENBASIS UND UNTERSUCHUNGSMETHODIK}

Trotz rechtlicher Restriktionen nimmt die Relevanz von Public Private Partnerships für die Praxis der öffentlichen Hand nicht ab. Welche Motive die unterschiedlichen Akteure bei der Implementation von PPP haben und inwieweit sie ihre Ziele erreichen, bleibt jedoch vor dem Hintergrund der vorgestellten quantitativen Daten offen. In den letzten Jahren wurden daher einige qualitative Forschungsprojekte durchgeführt, die typische Fallkonstellationen von PPP erfassen und auf dieser Basis Aufschluss über Motive und Wirkungen geben sollten.

Bei den in diesem Beitrag berücksichtigten Forschungsvorhaben handelt es sich um das Projekt „PPP - Hybridvarianten der Dienstleistungsproduktion", 1 die Untersuchung „PPP als neuartige Regelungsmuster zwischen öffentlicher Hand und Unternehmen“2 sowie das Vorhaben „BestPractice-Studie Projektfinanzierung und Öffentlich Private Partnerschaften in der Elektrotechnik- und Elektronikindustrie“.3

Im Zentrum standen Fragen danach, was der öffentliche Partner in PPP über den Umgang mit neu entstehenden oder veränderten Wettbewerbsbedingungen lernen kann, und nach den Wirkungen von PPP für die Nutzer öffentlicher Dienstleistungen (eine detaillierte Darstellung der qua- litativen Empirie findet sich bei Gerstlberger/Schröder 2008).

Es wurden Fälle untersucht, die in der politischen und fachlichen Öffentlichkeit als erfolgreiche Modelle für PPP gelten. Dafür wurden leitfadengestützte Experteninterviews mit politischen Entscheidern, Vertretern der öffentlichen Hand, Repräsentanten der privaten Partner, Personal- und Betriebsräten sowie ggf. weiteren relevanten Akteuren (z. B. Beratern) durchgeführt. Die qualitativen Ergebnisse wurden durch Dokumentenanalysen und Internetrecherchen ergänzt (Gerstlberger/Schneider 2008, S. 9f.).

\subsection{ZUSAMMENFASSUNG DER ERGEBNISSE QUALITATIVER FALLSTUDIEN}

Angesichts der Erwartung, PPP trüge zu Einsparungen oder Effizienzgewinnen bei, überrascht es, dass durchgängig in allen untersuchten Best-Practice-Fällen wirtschaftliche und finanzielle Aspekte nur am Rande eine Rolle spielen. Mindestens bei Projektbeginn stehen andere Ziele im Vordergrund. Einerseits ist dies die Vorbereitung auf Wettbewerbsbedingungen, mit welchen die öffentliche Hand teilweise erst Erfahrungen sammeln muss. Andererseits handelt es sich um Vorhaben, in denen die öffentliche Hand mittels externen Sachverstands Aufgaben bewältigen will, für die sie sich selbst aus politischen oder aus fachlichen Gründen nur begrenzt kompetent fühlt. Einsparungen sind eher Nebeneffekte. Strategisch wichtig ist der öffentlichen Hand oft, einen privaten Partner zu finden, der vertrauenswürdig ist und zur Organisationskultur der bestehenden bzw. zu schaffenden (teil-)öffentlichen Einrichtung passt. Private Partner akzeptieren ihrerseits, mit PPP nur begrenzt Gewinne machen zu können, um Referenzmodelle für künftige Partnerschaften und Vertrauenskapital aufzubauen.

Jenseits einfacher Dienstleistungen, bei denen die Gewinnspannen begrenzt sind,

\footnotetext{
Das Projekt wurde 2000 bis 2004 vom Bundesministerium für Bildung und Forschung (BMBF) durchgeführt. Es ist von Maria Oppen geleitet sowie von Friedrich Hauss, Detlef Sack, Karsten Schneider und Alexander Wegener bearbeitet worden.

2 Die Hans-Böckler-Stiftung förderte das Projekt von 2003 bis 2004 (Gerstlberger/Schmittel 2004).

3 Das Vorhaben erhielt 2005 und 2006 durch den Zentralverband Elektrotechnik- und Elektronikindustrie (ZVEI) e. V. Unterstützung (Gerstlberger et al. 2006).
} 
können beide Partner von einer dynamischen wirtschaftlichen Entwicklung profitieren und ihre Wettbewerbsposition durch die Kooperation teilweise erheblich verbessern. Aber auch wirtschaftlich erfolgreiche Partnerschaften entstehen in der Regel aus einer doppelten Notlage der beiden Partner. Der private Partner hat es entweder versäumt, sich in einem neuen Marktfeld frühzeitig zu positionieren, oder ist dem öffentlichen Partner aufgrund von langjähriger Interdependenz verpflichtet. Auf Seiten der öffentlichen Hand liegt oft ein strategisches Versäumnis - in der Regel ausgebliebene Investitionen oder Qualifikationsmaßnahmen - oder ein politisches Motiv vor.

Erfolge von Public Private Partnerships sind zumeist relativ unabhängig davon, $\mathrm{ob}$ es bei der Partnerschaft bleibt, der private Partner die Aufgabe komplett übernimmt oder die öffentliche Hand nach einiger Zeit wieder die Gesamtverantwortung erhält. Dies lässt die begründete Vermutung zu, dass PPP häufig eine Variante der betriebswirtschaftlichen Modernisierung des öffentlichen Partners ist. Oft arbeiten private Partner mit anderen Standards als die öffentliche Hand: In den Aufgabenfeldern Entsorgung, Gebäudemanagement oder ÖPNV bleibt man unter den Tarifentgelten des öffentlichen Dienstes; Gebühren und Preise für Ver- und Entsorgungsleistungen werden erhöht; die Qualität von Dienstleistungen, z. B. beim Catering, wird reduziert. Dem Privaten bleibt in der Regel wenig Spielraum für Kosteneinsparungen, wenn er derartige Standards - z. B. aufgrund vertraglicher Vereinbarungen - nicht senken kann. Für Beschäftigte bedeuten die strukturellen Veränderungen der Leistungserstellung, dass sie zunehmend mit ökonomischen Kriterien konfrontiert sind und Arbeitsprozesse verdichtet werden. Häufig sind die Beschäftigten und ihre Gewerkschaften von PPP eher betroffen als daran beteiligt.

Eine wichtige Erfolgsbedingung öffentlich-privater Partnerschaften ist eine gemeinsame positive Organisationskultur. Beschäftigte des privaten und öffentlichen Partners müssen friktionsfrei zusammenarbeiten. Dies ist bereits bei Fusionen von privaten Partnern nur selten gegeben. PPP sind diesbezüglich voraussetzungsvoller.
Zudem sind die Finanzierungsbedingungen der öffentlichen Hand relativ günstig und das Qualifikationsniveau ihrer Mitarbeiter ist gut, sodass kaum kleine und mittlere Unternehmen als Partner in Betracht kommen. Kommunen mit relativ schlechten Finanzierungsbedingungen sind darüber hinaus oft mit einem Veto der Kommunal- bzw. Finanzaufsicht konfrontiert, da PPP als kreditähnliche Geschäfte interpretiert werden.

Die öffentliche Hand ist im Rahmen von Partnerschaften dazu gezwungen, entweder effektiv zu steuern und sich mit den Eigeninteressen der PPP-Gesellschaft und des privaten Partners auseinanderzusetzen. Oder sie gibt die Kontrolle weitgehend aus der Hand. Ersteres impliziert erheblichen Ressourcenaufwand. Letzteres führt zur Abhängigkeit vom privaten Partner und zu dem Abbau eigener Kompetenz. In der Summe lässt sich der Aufbau von Vertrauen bzw. die Existenz von langfristig stabilen sozialen Beziehungen als Voraussetzung erfolgreicher PPP feststellen. Die angesichts hoher Transaktionskosten geforderten und durch Kompetenzzentren der Länder und des Bundes geförderten „PPP von der Stange“ sind insofern und angesichts dynamischer Wettbewerbsbedingungen realitätsfern. PPP können letztlich nur dann einen sinnvollen Zweck erfüllen, wenn sie beiden Partnern projektspezifische Lernerfolge ermöglichen.

\section{Fazit: PPP als begrenztes und spezialisiertes Finanzierungsinstrument}

Angesichts der Ergebnisse dieser Zwischenbilanz muten die Ziele, die in Wissenschaft und Politik mit PPP verbunden werden, überraschend an. Von besserem Regierungshandeln und selbst finanziellen Einsparmöglichkeiten ist in der Praxis kaum die Rede. Den öffentlichen Partnern geht es in Best-Practice-Fällen in erster Linie um neue Finanzierungsmöglichkeiten sowie die Einwerbung von wettbewerblichem und technischem Know-how. Entscheidende Rahmenbedingung von PPP ist, dass die Haushaltsengpässe der öffentlichen Hand
Investitionen in die Infrastruktur kaum mehr möglich machen. PPP sind demnach eine Option, trotz fehlender weiterer Verschuldungsmöglichkeiten Ressourcen für öffentlich zu erbringende Dienstleistungen $\mathrm{zu}$ mobilisieren. Dabei handelt es sich nicht nur um finanzielle Mittel, sondern auch um Qualifizierungsmöglichkeiten und wettbewerbsrelevantes Wissen, welches sich die öffentliche Hand nicht kurzfristig beschaffen kann.

Die qualitative Empirie macht auch deutlich, dass die von den öffentlichen Partnern formulierten Ziele grundsätzlich erreicht werden können. Allerdings ist dies an Voraussetzungen gebunden. Förderlich für den Erfolg ist in erster Linie die von beiden akzeptierte gegenseitige Abhängigkeit der Partner. Diese existiert nicht nur aufgrund der Partnerschaften selbst, sondern auch durch vorgelagerte Kooperationsbeziehungen (z. B. Auftraggeber-Auftragnehmer- bzw. Kundenbeziehungen). Zudem sind private Partner auch über das jeweilige Projekt hinaus in hohem Maße am langfristigen Erfolg von PPP interessiert. Es sollen immer auch Referenzmodelle für künftige Partnerschaften entwickelt werden.

Das Problem des Vertrauensaufbaus, das für erfolgreiche PPP von entscheidender Bedeutung ist, erscheint derzeit ungelöst. In der Vergangenheit hat es in aller Regel vertrauensvolle Partnerschaften zwischen Privaten und Öffentlichen gegeben, sofern stabile Interaktionsbeziehungen und Abhängigkeiten bereits bestanden. In Zukunft ist keine (kurzfristige) Lösung für das Problem des Vertrauensaufbaus zu erwarten, da die Rechtsprechung des EuGH dem entgegensteht. Sollte es zu keiner Neufassung bzw. -interpretation des Ausschreibungsrechts seitens der EU kommen, verliert PPP als Alternative zu materieller Privatisierung an Bedeutung. Die Ausschreibungspflicht bei Inhouse-Geschäften zwischen Kommunen und ihren Eigengesellschaften verhindert oder erschwert zumindest den Aufbau dauerhafter Vertrauensbeziehungen zwischen öffentlichen und privaten Partnern. Public Private Partnership bleibt damit ein begrenztes und spezialisiertes Finanzierungsinstrument, dessen Chancen und Risiken im Einzelfall abzuwägen sind. 
Bayerischer Oberster Rechnungshof (ORH) (2006): Jahresbericht 2006, München

Bogumil, J./Grohs, S./Kuhlmann, S./Ohm, A. K. (2007): Zehn Jahre Neues Steuerungsmodell. Eine Bilanz kommunaler Verwaltungsmodernisierung, Berlin

Budäus, D. (Hrsg.) (2006): Kooperationsformen zwischen Staat und Markt. Theoretische Grundlagen und praktische Ausprägungen von Public Private Partnership, Baden-Baden

Christen, J. (2007): ÖPP-Leitfaden, Berlin

Deutsches Institut für Urbanistik (Difu) (2008): PPP und Mittelstand. Untersuchung von 30 ausgewählten PPP-Hochbauprojekten in Deutschland, Berlin

Edeling, T./Stölting, E./Wagner, D. (2004): Öffentliche Unternehmen zwischen Privatwirtschaft und öffentlicher Verwaltung. Eine empirische Studie im Feld kommunaler Versorgungsunternehmen, Wiesbaden Ellwein, T./Buck, L. (1995): Wasserversorgung - Abwasserbeseitigung: öffentliche und private Organisation, Landsberg

Europäischer Gerichtshof (EuGH) (2005): Stadt Halle, RPL/Recyclingpark Lochau $\mathrm{GmbH}$ gegen Arbeitsgemeinschaft Thermische Restabfall- und Energieverwertungsanlage TREA Leuna, Entscheidungssammlung des EuGH, AZ: C-26/03, Entscheidung vom 11.01., Brüssel

EUROSTAT (2004): EUROSTAT-Entscheidung zu PPP vom 11.02., Brüssel, download: http://epp.eurostat.cec.eu.int/cache/ITY_PUBLIC/ 2-11022004-AP/DE/2-11022004-AP-DE.HTML, 17.03.2007 Frischmuth, B. (2004): Kompetenzzentren aus Sicht des Deutschen Städtetages, in: Knop, D. (Hrsg.): Public Private Partnership. Jahrbuch 2004, Wiesbaden, S. 42-46

Gerstlberger, W./Höhne, W./Siegl, M. (2006): Best-Practice-Studie Projektfinanzierung und Öffentlich Private Partnerschaften (ÖPP) im Markt der Elektrotechnik- und Elektronikindustrie, Frankfurt/Main Gerstlberger, W./Sack, D. (2003): Public Private Partnership für E-Government: Internationale Recherche, Praxisleitfaden und Fallbeispiele, Gütersloh

Gerstlberger, W./Schmittel, W. (2004): Public Private Partnership als neuartiges Regelungsmuster zwischen öffentlicher Hand und Unternehmen, Düsseldorf

Gerstlberger, W./Schneider, K. (2008): Öffentlich Private Partnerschaften. Zwischenbilanz, empirische Befunde und Ausblick, Berlin Grabow, B./Reidenbach, M./Rottmann, M./Seidel-Schulze, A. (2005): Public Private Partnership Projekte - Eine aktuelle Bestandsaufnahme in Bund, Ländern und Kommunen, Berlin

Gesprächsrunde PPP (2006): PPP - Public Private Partnership zur Realisierung öffentlicher Baumaßnahmen in Bayern, München Institute for Public Policy Research (IPPR) (2001): Building Better Partnerships, London
Janetschek, M. (2007): Privatisierung und ÖPP als Ausweg? Kommunalfinanzen unter Druck - Handlungsoptionen für Kommunen, Eschborn Killian, W./Richter, P./Trapp, J. H. (2006): Ausgliederung und Privatisierung in Kommunen. Empirische Befunde zur Struktur kommunaler Aufgabenwahrnehmung, Berlin

Konferenz der Präsidentinnen und Präsidenten der Rechnungshöfe des Bundes und der Länder (Hrsg.) (2006): Gemeinsame Position der Rechnungshöfe aus Bund und Ländern zu PPP vom Mai 2006, Berlin Kiefer, D. (2006): "Chancen und Risiken von PPP-Projekten der 1. und 2. Generation aus Sicht des Rechnungshofs Baden-Württemberg, Vortrag im Rahmen der Tagung Public Private Partnership bei Bau und Betrieb von Einrichtungen der Wissenschaft, Mannheim, 2. bis 4. Juni 2005, Stuttgart (unveröffentlichtes Manuskript)

Kruzewicz, M. (1993): Lokale Kooperationen in NRW, Public Private Partnership auf kommunaler Ebene, Düsseldorf

Littwin, F./Schöne, F.-J. (Hrsg.) (2006): Public Private Partnership im öffentlichen Hochbau, Stuttgart

Napp, H.-G. (2004): PPP im Entsorgungsbereich - Zusammenarbeit zwischen öffentlicher Hand, privaten Dienstleistern, Investoren und finanzierenden Banken, in: GöW (Hrsg.): Public Private Partnership: Formen, Risiken, Chancen, Baden-Baden, S. 149-168

Sack, D. (2007): Governance und Politics - Die Institutionalisierung öffentlich-privater Partnerschaften in Deutschland (Habilitationsschrift), Kassel

Schneider, K. (2002): Arbeitspolitik im „Konzern Stadt“. Zwischen der Erosion des Zusammenhalts im kommunalen Sektor und den effizienzfördernden Wirkungen organisatorischer Dezentralisierung, Baden-Baden SPD-Bundestagsfraktion (2004): Öffentlich Private Partnerschaften. Neue Chancen für öffentliche Leistungen, Berlin Sühnel, S. (2008): Zur Anwendung des europäischen Beihilferechts auf kommunale Unternehmen, Berlin

Trapp, J. H. (2006): Ausgliederung und Privatisierung in den dreißig größten deutschen Städten, in: Killian, W./Richter, P./Trapp, J. H. (Hrsg.): Ausgliederung und Privatisierung in Kommunen. Empirische Befunde zur Struktur kommunaler Aufgabenwahrnehmung, Berlin, S. 85-109 Weber, M./Schäfer, M./Hausmann, F. L. (Hrsg.) (2006): Praxishandbuch Public Private Partnership, München

Wegener, A. (2002): Die Gestaltung des kommunalen Wettbewerbs. Strategien in den USA, Großbritannien und Neuseeland, Berlin Weizsäcker, E. U. von/Young, O. R./Finger, M. (2006): Grenzen der Privatisierung. Wann ist des Guten zuviel? Bericht an den Club of Rome, Stuttgart

Ziekow, J./Windoffer, A. (2008): Public Private Partnership. Struktur und Erfolgsbedingungen von Kooperationsarenen, Baden-Baden 